\title{
Introduction du frottement sur les dentures dans la simulation du comportement dynamique de transmissions par engrenages
}

\author{
Fabrice Ville ${ }^{\mathrm{a}}$ et Philippe Velex \\ Laboratoire de Mécanique des Contacts et des Structures, LaMCoS, INSA-Lyon, CNRS UMR 5259, \\ 69621 Villeurbanne Cedex France
}

Reçu le 15 mars 2007, accepté le 7 juin 2007

\begin{abstract}
Résumé - Un modèle du comportement dynamique de trains d'engrenages incluant les effets du frottement sur les dentures est présenté. Les résultats de ce modèle confrontés aux mesures obtenues sur banc d'essai montrent un très bon accord. Des simulations ont été réalisées sur de larges plages de vitesses de rotation pour différentes corrections de denture afin d'analyser les contributions spécifiques du frottement.
\end{abstract}

Mots clés : Frottement / denture / engrenages / dynamique

\begin{abstract}
A model of the dynamic behaviour of spur gear transmissions including the effects of tooth friction is presented. The results of this model confronted with the measurements obtained on a specific test bench show a very good agreement. Simulations were carried out for various tooth profile modifications in order to analyze the specific contributions of friction.
\end{abstract}

Key words: Friction / teeth / gears / dynamics

\section{Introduction}

La très large majorité des modélisations traitant de la dynamique des engrenages néglige les contributions des efforts de frottement entre les dentures et considère que les effets normaux aux contacts sont prépondérants en termes d'excitation : Özgüven et Houser (1988) [1] et Blankenship et Singh (1992) [2]. Toutefois, des résultats expérimentaux récents ont remis partiellement en cause cette hypothèse fondamentale pour ce qui concerne les efforts instantanés transmis par les paliers et donc la génération de bruit par rayonnement du carter : Börner et Houser (1996) [3] et Velex et Cahonet (2000) [4]. Dans cet article, un modèle tridimensionnel d'engrenages simple étage incluant les contributions du frottement est présenté. Le pignon et la roue sont assimilés à des cylindres rigides liés par des distributions de rigidités dépendantes du temps distribuées selon les lignes de contact potentielles. Chaque ligne de contact est discrétisée en cellules indépendantes auxquelles sont affectés : a) une rigidité élémentaire, b) un écart normal équivalent afin de tenir compte de corrections de forme ou d'erreurs géométriques, c) un coefficient de frottement et par conséquent un effort tangent au profil de denture. Au cours du mouvement, les positions des

\footnotetext{
${ }^{a}$ Auteur correspondant : fabrice.ville@insa-lyon.fr
}

lignes de contact évoluent sur les flancs des dentures et les caractéristiques de discrétisation sont réactualisées en fonction de ces évolutions. Les arbres sont simulés par des éléments-finis en flexion, torsion et traction-compression à deux nœuds et six degrés de liberté par nœud. Enfin, les paliers sont modélisés par des raideurs concentrées. Les résultats de simulation sont ensuite comparés à des résultats expérimentaux relevés sur un banc d'engrenages fortement instrumenté : Velex et Cahouet (2000) [4] et Baud et Velex (2002) [5]. L'importance potentielle du frottement sur le transfert de vibration aux paliers et par conséquent sur le niveau de bruit généré par une transmission est mise en évidence. Une analyse paramétrique sur l'influence couplée de corrections de profil et du frottement conclut cet article.

\section{Aspects expérimentaux}

Le banc d'engrenages utilisé pour la validation du modèle de comportement dynamique avec frottement est un train simple étage avec dentures droites et paliers hydrostatiques (l'ensemble des caractéristiques et des configurations possibles est décrit dans Baud (1998) [6]). Les caractéristiques géométriques des engrenages sont portées tableau 1, les dentures sont rectifiées (classe ISO 4) et les 
Tableau 1. Données engrenages sur banc d'essai.

\begin{tabular}{lcc}
\hline & \multicolumn{2}{c}{ Dentures droites } \\
\hline Module $(\mathrm{mm})$ & \multicolumn{3}{c}{4} \\
\hline & pignon & roue \\
Nombre de dents & 27 & 156 \\
Largeur (mm) & 50 & 40 \\
Angle de pression $\left(^{\circ}\right)$ & \multicolumn{2}{c}{20} \\
Coefficient de creux & \multicolumn{2}{c}{1,4} \\
Coefficient de saillie & \multicolumn{2}{c}{1,0} \\
Coefficient de déport & 0,16 & $-0,16$ \\
\hline
\end{tabular}

profils des dents du pignon et de la roue sont corrigés (amplitude en tête de $20 \mu \mathrm{m}$ sur une longueur correspondant à $20 \%$ du profil actif des dents). La lubrification forcée se fait par deux jets au voisinage de l'engrènement avec de l'huile de relativement faible viscosité (ISO VG 60).

Les forces de frottement sur les dentures sont déduites des efforts mesurés aux paliers par des tables dynamométriques tridimensionnelles et projetées dans la direction normale au plan d'action (soit la direction tangente aux profils de denture). Les efforts de frottement mesurés ne correspondent pas aux efforts sur un contact mais sont les efforts totaux représentant les contributions de tous les contacts à un instant donné.

\section{Modèle du comportement dynamique d'engrenages}

\section{1 État de référence}

Les mouvements de corps rigides définissent l'état de référence. La cinématique, indépendante du frottement, est contrôlée par le rapport de transmission entre le pignon et la roue ainsi que par les écarts de forme éventuels induisant une erreur de transmission sans charge variable au cours du temps : Velex et Maatar (2000) [7]. Dans ce qui suit, la vitesse sur le pignon est supposée constante. La dynamique de l'état de référence, quant à elle, dépend $\mathrm{du}$ frottement sur les dentures.

\section{2 État déformé}

Les petits déplacements généralisés au voisinage des mouvements de corps rigides sont décrits par des torseurs de déplacements infinitésimaux (3 translations et 3 rotations) en tout noud.

Le point central de la modélisation concerne la description des efforts normaux et tangentiels sur les contacts entre dents. Les rigidités d'engrènement permettent de simuler les effets normaux tandis que les efforts de frottement en sont déduits par la loi de Coulomb. On note que selon la position de la ligne de contact, le sens du glissement et, par conséquent la direction de l'effort de frottement, sont susceptibles de changer au cours du mouvement.
L'application des équations de Lagrange conduit à un système différentiel à coefficients dépendants du temps et éventuellement non-linéaire suite aux interactions entre les aires de contact (et, par conséquent, la rigidité de la liaison d'engrènement) et les degrés de liberté. Les équations du mouvement peuvent être écrites sous la forme :

$$
\begin{aligned}
{[M]\{x\}+[C]\{\ddot{x}\} } & +[K(t, x)]\{\dot{x}\} \\
+ & \{F\}+\left\{F_{\text {frot }}(f, t, x)\right\}+\left\{F_{\text {écarts }}(t, x)\right\}
\end{aligned}
$$

La résolution du système ne peut être conduite que de manière itérative et combine un schéma d'intégration pas à pas avec des algorithmes qui vérifient que les efforts normaux de contact sont compressifs, qu'il n'y a de déflexion (ou d'interférence) ailleurs que dans la zone de contact et, enfin, qu'il y a convergence sur les efforts de frottement.

Les détails sur les aspects mathématiques et numériques associés à la modélisation peuvent être trouvés dans Velex et Maatar (1996) [7].

\section{Comparaisons entre résultats expérimentaux et simulations numériques}

Le comportement du banc d'engrenages a été simulé et les résultats pour trois valeurs de coefficients de frottement (respectivement $0,0,05$ et 0,1 ) et deux vitesses de rotation (200 et $600 \mathrm{tr} \cdot \mathrm{min}^{-1}$ sur le pignon) sont portés figure 1. L'effort de frottement total instantané (c'est-à-dire l'effort dans la direction normale au plan d'action) est porté selon l'axe vertical, l'axe horizontal représente le temps adimensionné par rapport à la période d'engrènement.

Les principales observations sont :

a. Un rôle a priori non négligeable du frottement à la denture sur les efforts transmis aux paliers. Dans le cas où le frottement est négligeable, l'effort statique au palier est constant alors que les résultats de mesure montrent clairement des fluctuations importantes de cet effort.

b. Des fluctuations de frottement sont observées i) au passage du primitif lorsque la vitesse de glissement change de signe et ii) pour la vitesse de rotation la plus faible suite sans doute aux conditions de lubrification particulière à ce régime (lubrification limite).

c. Un bon accord général entre les résultats expérimentaux et théoriques en particulier pour $f=0,05$ qui correspond à une valeur habituelle dans la littérature. Ce constat permet donc de très largement valider le modèle de frottement et son intégration dans des simulations de transmissions par engrenages.

\section{Exploitations paramétriques}

Une série de calculs a été réalisée pour des engrenages définis tableau 2. L'objectif principal est de vérifier si les 


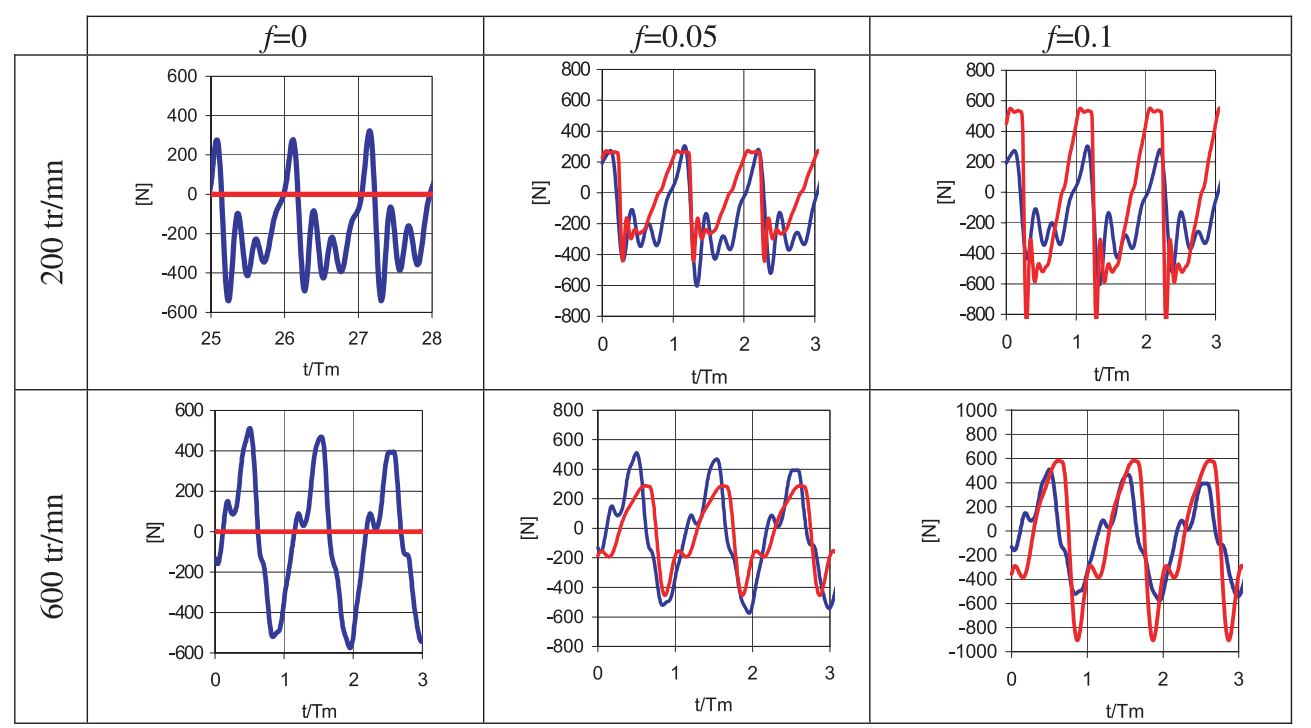

Fig. 1. Efforts de frottement mesurés (en bleu) et simulés (en rouge). Dentures droites.

Tableau 2. Données engrenages pour exploitations paramétriques.

\begin{tabular}{|c|c|c|}
\hline & \multicolumn{2}{|c|}{ Dentures droites } \\
\hline Entraxe $(\mathrm{mm})$ & \multicolumn{2}{|c|}{63} \\
\hline \multirow[t]{2}{*}{ Module (mm) } & \multicolumn{2}{|c|}{2,1} \\
\hline & pignon & roue \\
\hline Nombre de dents & 24 & 36 \\
\hline Largeur (mm) & 48 & 44 \\
\hline Angle de pression $\left({ }^{\circ}\right)$ & \multicolumn{2}{|c|}{25} \\
\hline Coefficient de creux & \multicolumn{2}{|c|}{1,4} \\
\hline Coefficient de saillie & \multicolumn{2}{|c|}{1,0} \\
\hline Coefficient de déport & 0,0 & 0,0 \\
\hline
\end{tabular}

tendances observées en régime quasi-statique peuvent être étendues au comportement à grandes vitesses de rotation.

Les efforts dynamiques sur l'engrènement en fonction de la vitesse de rotation sont portés sur la figure 2 et apparaissent comme très largement indépendant du frottement sur les dentures. En revanche, les efforts dynamiques transmis aux paliers (Fig. 3) sont très sensibles au frottement qui, en particulier, fait émerger des pics de résonance importants.

Des corrections de profil symétriques en tête des dents du pignon et de la roue sont introduites dans les simulations. La profondeur de correction en tête de dent est de $30 \mu \mathrm{m}$ pour une longueur correspondant à $30 \%$ du profil actif des dentures. Les efforts dynamiques à l'engrènement représentés figure 4 sont considérablement atténués et demeurent insensibles au niveau de frottement sur les dents. De manière intéressante, les efforts dynamiques aux paliers (Fig. 5), quant à eux, ne sont pas réduits de manière significative par les corrections de forme. L'allure des courbes de réponse est différente mais les amplitudes maximales sont, pour certaines vitesses, supérieures à celles relevées pour des dentures non corrigées.

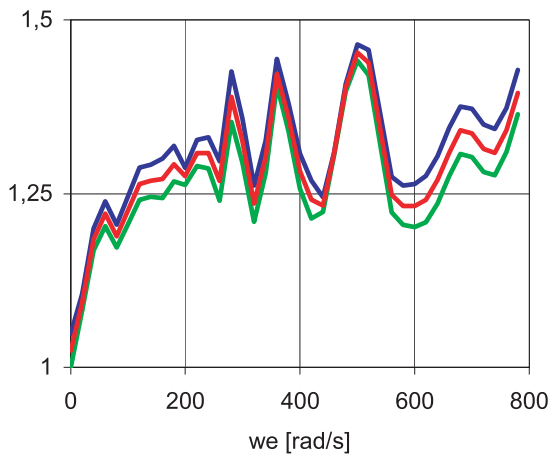

Fig. 2. Effort dynamique à la denture adimensionné par rapport à l'effort statique en fonction de la vitesse du pignon. ( $f=0$, vert ; $f=0,05$, rouge; $f=0,1$, bleu).

Ces observations suggèrent un relatif découplage entre :

a. les effets normaux aux contacts qui conditionnement très largement le chargement sur les dentures,

b. les effets tangentiels liés au frottement qui influencent fortement les efforts transmis aux paliers et qui, par conséquent, vont jouer un rôle potentiellement non négligeable sur la génération de bruit par rayonnement du carter.

Une des conséquences pratiques est que l'optimisation de corrections de forme sur les dentures afin de réduire les excitations vibratoires pourrait s'avérer peu efficace en terme de bruit généré suite à l'influence du frottement décrite plus haut.

\section{Conclusions}

Un modèle du comportement dynamique tridimensionnel d'engrenages incluant l'influence du frottement 


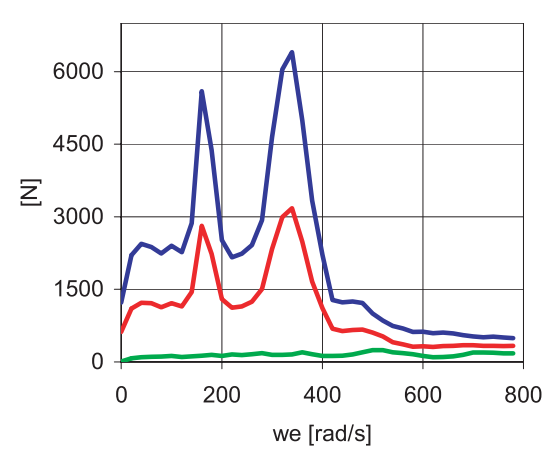

a- direction de la ligne des centres des engrenages



\section{b- direction perpendiculaire à la ligne des centres des engrenages}

Fig. 3. Fluctuation d'effort au palier ( $f=0$, vert; $f=0,05$, rouge; $f=0,1$, bleu).

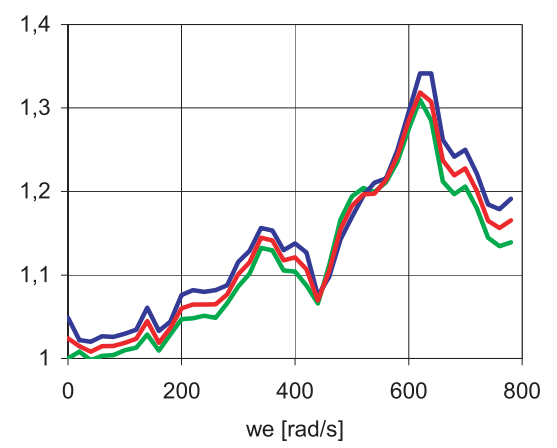

Fig. 4. Effort dynamique à la denture adimensionné par rapport à l'effort statique en fonction de la vitesse du pignon. Dentures droites corrigées $(f=0$, vert; $f=0,05$; rouge; $f=0,1$, bleu).

sur les dentures est présenté. Le frottement sur les dentures est intégré par une loi locale de comportement de type Coulomb, tandis que les effets normaux aux contacts sont décrits par des raideurs d'engrènement et des écarts équivalents distribués sur les lignes de contact potentielles entre le pignon et la roue. La résolution est conduite pas à pas dans le temps en combinant des algorithmes de contact normaux et tangentiels. Les résultats de simulation sont en bon accord avec des résultats expérimentaux

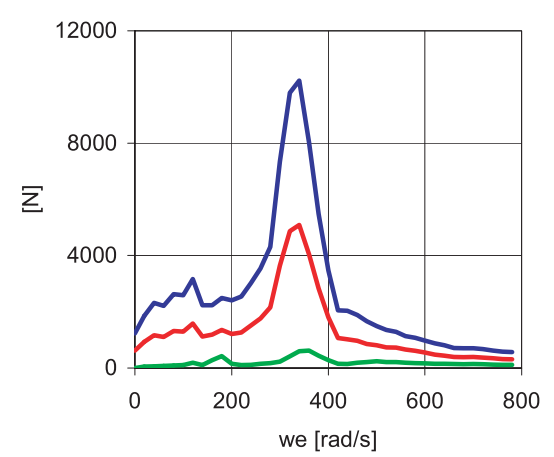

a- direction de la ligne des centres des engrenages

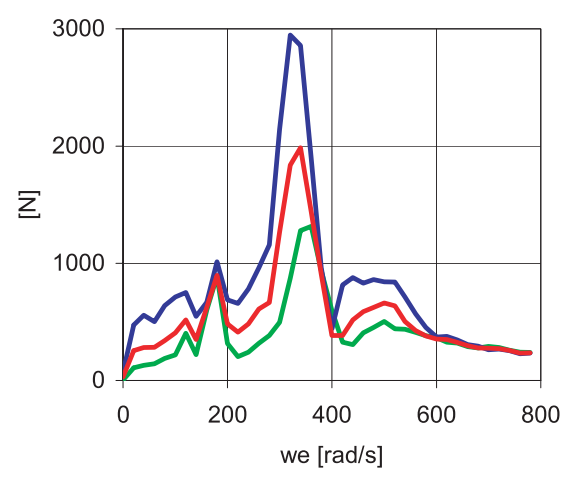

\section{b- direction perpendiculaire à la ligne des centres des engrenages}

Fig. 5. Fluctuation d'effort au palier. Dentures droites corrigées ( $f=0$, vert; $f=0,05$, rouge; $f=0,1$, bleu).

obtenus à basses vitesses de rotation sur banc d'engrenages. Les principaux constats sont :

a. une influence négligeable du frottement sur le chargement des dentures,

b. une influence marquée sur les fluctuations d'efforts transmis par les paliers et, par conséquent, sur le niveau de bruit généré par transmission solidienne

c. l'apport de corrections de profil sur le chargement des dentures est confirmé, par contre on ne note pas d'amélioration notable au niveau des efforts dynamiques induits par frottement aux paliers.

\section{Références}

[1] H.N. Özgüven, D.R. Houser, Mathematical models used in gear dynamics - A review, J. Sound and Vibration 121 (1988) 383-411

[2] G.W. Blankenship, R. Singh, A comparative study of selected gear mesh interface dynamic models, Proceedings of the ASME 6th International Conference on Power Transmissions and Gearing, Phoenix, 1992, pp. 137-146

[3] J. Börner, D.R. Houser, Influence of friction and bending moments on gear noise excitations, SAE Transactions 105 (1996) 1669-1676 
[4] P. Velex, V. Cahouet, Experimental and numerical investigations on the influence of tooth friction in spur and helical gear dynamics, ASME J. Mechanical Design 122 (2000) 515-522

[5] S. Baud, P. Velex, Static and dynamic tooth loading in spur and helical geared systems - Experiments and model validation, ASME J. Mechanical Design 124 (2002) 334346
[6] S. Baud, Développement et validation sur banc d'essais de modèles du comportement dynamique de réducteurs à engrenages à axes parallèles, thèse de Doctorat, INSA de Lyon, 194 p. 1998

[7] P. Velex, M. Maatar, A mathematical model for analyzing the influence of shape deviations and mounting errors on gear dynamic behaviour, J. Sound and Vibration 191 (1996) 629-660 